\title{
ANÁLISE COMPARATIVA DE CARGAS DE LAMINAÇÃO A QUENTE INDUSTRIAIS COM AS OBTIDAS ATRAVÉS DE MODELAMENTO MATEMÁTICO
}

\author{
Evaldo Diniz Dias ' \\ Jose Adilson de Castro ' \\ Fabio de Oliveira Araújo ${ }^{2}$ \\ Carlos Roberto Xavier ${ }^{3,4}$ \\ Alexandre Pimentel Sampaio ${ }^{2}$
}

\section{Resumo}

Uma análise comparativa entre as cargas de laminação calculadas através dos modelos de Sims, Orowan-Pascoe e Ekelund é realizada a fim de verificar o melhor desempenho, frente aos valores registrados durante o processamento de aços C-Mn e ao nióbio em um trem acabador de tiras a quente industrial. Para determinação da tensão média de escoamento são utilizadas as equações de Shida, Poliak \& Siciliano, Siciliano, Marini \& Bruna, Siciliano, Leduc \& Hensger, Misaka e Siciliano \& Jonas. Na comparação direta entre os valores calculados e os reais, os melhores níveis de precisão são obtidos quando os modelos de previsão de carga são combinados a equação de Shida, respectivamente Sims, Orowan-Pascoe e Ekelund. Neste trabalho são propostos fatores de correção visando obter maior previsibilidade para o processo industrial durante a laminação de acabamento. Com a aplicação dos fatores de ajuste as equações de previsão de carga apresentaram erros médios globais no trem de acabamento, variando entre 4,3 e 5,6\% para os aços C-Mn e 4,6 e 6,3\% para aços microligados ao nióbio.

Palavras-chave: Laminação a quente; Cargas; Modelamento matemático.

\section{COMPARATIVE ANALYSIS OF INDUSTRIAL HOT ROLLING LOADS AND THOSE OBTAINED THROUGH MATHEMATICAL MODELING}

\begin{abstract}
A comparison between the rolling loads calculated through the theoretical models of Sims, Orowan-Pascoe and Ekelund is performed in order to verify the performance of the models compared to the real values recorded during the processing of plain carbon-manganese steels and niobium steels during the finish rolling in the hot strip mill. To determine the hot deformation resistance, the equations of Shida, Poliak \& Siciliano, Siciliano, Marini \& Bruna, Siciliano, Leduc \& Hensger, Misaka and Siciliano \& Jonas are used. In the direct comparison between the calculated values and the actual values the best level of precision is obtained when the load prediction models are combined with the Shida equation, respectively Sims, Orowan-Pascoe and Ekelund. In order to better adjust calculated loads to the reality of the industrial process, adjustment factors are proposed for each of the seven stands of the finishing mill. With the application of the adjustment factors, the load prediction equations presented global mean errors in the finishing mill, varying between 4.3 and $5.6 \%$ for C-Mn steels and 4.6 and $6.3 \%$ for niobium steels.
\end{abstract}

Keywords: Hot rolling; Loads; Mathematical modeling.

\footnotetext{
'Programa de Pós-graduação em Engenharia Metalúrgica e de Materiais, Escola de Engenharia Industrial Metalúrgica de Volta Redonda, Universidade Federal Fluminense - UFF, Volta Redonda, RJ, Brasil. E-mail: evaldodiniz@id.uff.br

${ }^{2}$ Companhia Siderúrgica Nacional, Volta Redonda, RJ, Brasil.

${ }^{3}$ Departamento de Engenharia Mecânica, Centro Universitário de Volta Redonda - UniFOA, Volta Redonda, RJ, Brasil.

${ }^{4}$ PETROBRAS, Rio de Janeiro, RJ, Brasil.
}

2176-1523 (C) 2019 Associação Brasileira de Metalurgia, Materiais e Mineração. Publicado pela ABM. Este é um artigo de acesso aberto distribuído sob os termos da licença Creative Commons CC BY-NC-ND (Attribution-NonCommercial-NoDerivs) - https:// creativecommons.org/licenses/by-nc-nd/4.0\%. 


\section{INTRODUÇÃO}

O aperfeiçoamento do controle no processamento termomecânico de chapas de aço tem se tornado ponto crucial para a indústria siderúrgica, devido à crescente demanda de produtos com especificações cada vez mais rigorosas impostas pelos usuários finais. Como por exemplo, a indústria automotiva que pertence a um dos segmentos do mercado global que criam demandas contínuas de aperfeiçoamento de materiais, buscando aços com maiores requisitos de resistência mecânica ao passo que devem conservar elevados níveis de conformabilidade. O conhecimento prévio do comportamento das cargas de laminação é um fator extremamente importante para o controle de processo, garantia dos requisitos dimensionais e das propriedades mecânicas dos produtos planos.

Nas últimas décadas diferentes pesquisadores têm desenvolvido modelos matemáticos que tornam possível a previsão dos esforços na laminação a quente [I].

Orowan [2] foi pioneiro em desenvolver um modelo compressivo baseado na extensão do método de blocos, capaz de realizar o cálculo dos esforços de laminação, introduzindo a deformação elástica dos rolos e as deformações plásticas da tira. Posteriormente Orowan-Pascoe propuseram em conjunto uma equação simplificada para o cálculo da carga de laminação, considerando a condição de atrito por agarramento [3], conforme Equação I. Onde a carga de laminação pode ser calculada por unidade de largura:

$$
\frac{\mathrm{P}}{\mathrm{W}}=\bar{\sigma} \mathrm{LQ}_{\mathrm{p}}
$$

A força de laminação $\mathbf{P}$ através da relação entre a tensão média de escoamento $\bar{\sigma}$, a largura da tira $\mathbf{W}$, o arco de contato formado entre a tira e o cilindro de laminação $\mathbf{L}$ e um fator geométrico $Q_{p}$ [4]. Equações similares foram postuladas por Sims e Ekelund, a equação simplificada de Sims considera que a velocidade da tira e o cilindro de laminação são idênticas em todo o comprimento do arco de contato [5]. A equação Ekelund considera que na entrada do arco de contato a velocidade da tira é menor que a velocidade de rotação do cilindro, aumentando gradativamente até alcançar uma velocidade superior à do cilindro na saída do arco de contato [6].

O cálculo das cargas de laminação é sensivelmente influenciado por fatores como a resistência a deformação a quente e por mecanismos de restauração como recristalização estática, dinâmica e metadinâmica que podem ser ativados durante a laminação de acabamento [ $I$ ]. Estão disponíveis na literatura diferentes tipos de modelos para previsão da tensão média de escoamento. De acordo com Hodgson [7] estes modelos podem ser categorizados em: fenomenológicos, empíricos, semi-empíricos e heurísticos. Os modelos mais famosos são Misaka e Yoshimoto [8], Shida [9], Kirihata et al. [10], Poliak e Siciliano [I I] e Siciliano et al. [12], que apresentam a tensão como uma função da quantidade de deformação, taxa de deformação e da temperatura [13].

Para a realização do cálculo da tensão de escoamento o modelo de Shida leva em consideração a região de transformação da austenita para ferrita, o que reduz consideravelmente a resistência a deformação a quente do material [14]. O modelo de Shida é baseado em dados experimentais obtidos em teste de compressão em elevadas taxas de deformação [15]. No presente trabalho são realizadas análises comparativas entre cargas de laminação calculadas através dos modelos teóricos propostos por Sims, Ekelund e Orowan-Pascoe e as registradas durante o processamento industrial de aços C-Mn e aços microligados ao nióbio.

\section{MATERIAIS E MÉTODOS}

Para realização deste trabalho foram utilizados como fonte de dados os registros obtidos em um laminador de tiras a quente industrial. Na Figura I é apresentado da direita para a esquerda um desenho esquemático do laminador industrial.

O laminador de tiras a quente em questão é composto por 4 fornos de reaquecimento de placas, um laminador vertical (VE-I), um laminador duo-horizontal (R-I), um laminador quádruo universal reversível (VE-2/R-2) e dois laminadores quádruos universais combinados a dois laminadores verticais (VE-3/R-3 e VE-4/R-4). O trem acabador possui sete cadeiras de laminação (F-I a F-7), alcançando velocidade de até $I 308 \mathrm{mpm}$ na última cadeira, seguidos da mesa de saída e três bobinadeiras (DC-I, DC-2 e DC-3).

As informações coletadas incluem a composição química, características dimensionais da bobina (largura, comprimento e espessura), parâmetros de laminação dos passes de desbaste e acabamento (espessura, largura, temperatura de laminação, tempos entre passes, força de laminação, diâmetro e rotação dos cilindros).

Para os aços ao C-Mn a faixa de concentração de carbono foi mantida entre $0,02 \%$ e $0,065 \%$ e a de manganês entre $0,21 \%$ e $0,37 \%$. Para os aços microligados ao nióbio a faixa de concentração de carbono foi mantida entre $0,01 \%$

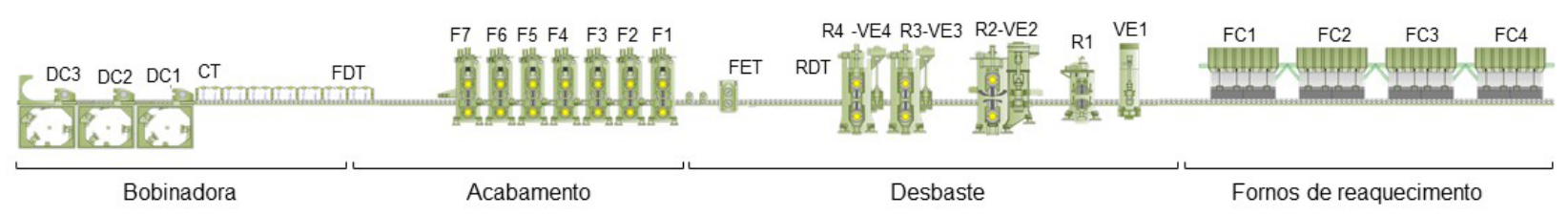

Figura I. Representação esquemática do laminador industrial. 
e $0,07 \%$, manganês entre $0,49 \%$ a $0,63 \%$ e nióbio entre $0,02 \%$ e $0,034 \%$. Foram analisadas um total de 2676 bobinas referentes aos aços C-Mn e aços microligados ao nióbio.

A partir dos dados registrados no processamento industrial, são utilizadas as equações simplificadas de Sims, Orowan-Pascoe e Ekelund para a realização da previsão das cargas de laminação para cada um dos sete passes do laminador de acabamento. $O$ cálculo da tensão média de escoamento (TME) foi realizado utilizando as equações de Shida, Siciliano, Marini \& Bruna, Poliak \& Siciliano, Siciliano, Leduc \& Hensger, Misaka e a equação de Misaka modificada por Siciliano e Jonas [16] contabilizando o efeito da concentração de manganês.

$\mathrm{Na}$ Tabela I são apresentadas as equações utilizadas, onde a concentração dos elementos $[x]$ é expressa em percentual de peso, $T$ a temperatura em Kelvin, $\&$ quantidade de deformação e $\dot{\varepsilon}$ a taxa de deformação $\left(\mathrm{s}^{-1}\right)$.

Para todas as equações apresentadas na Tabela I é considerada a completa recristalização entre passes. Entretanto na laminação de aços com a adição de nióbio a recristalização é frequentemente realizada de forma parcial, devido aos curtos intervalos de tempo entre os passes no trem acabador, quando as temperaturas são relativamente baixas [18]. Estes fatores contribuem para o acúmulo de deformação entre passes e favorece a ocorrência da recristalização dinâmica seguida de recristalização metadinâmica.

Durante o cálculo das cargas de laminação a condição de atrito por agarramento é considerada para os modelos de Sims e Orowan-Pascoe, já o modelo de Ekelund utiliza o critério de atrito por deslizamento. O coeficiente de atrito por deslizamento foi calculado utilizando a equação de Roberts [19].

Os arranjos realizados entre as equações para cálculo das cargas de laminação e os modelos para previsão da tensão média de escoamento totalizaram $2 \mathrm{I}$ abordagens. O grupo de 2676 dados referentes às bobinas processadas no laminador industrial foi dividido em dois subgrupos: aços C-Mn com 244 bobinas e aços microligados ao nióbio com 2432 bobinas. A análise dos resultados obtidos através das combinações realizadas entre os modelos teóricos e as cargas de laminação registradas no processamento industrial são realizadas inicialmente de forma direta, ou seja, sem a introdução de fatores de ajustes.

Posteriormente são realizadas novas comparações adotando fatores de correção, com objetivo de identificar os melhores arranjos de acordo com o menor erro padrão entre as cargas calculadas e as registradas na planta industrial. A Equação 2 é utilizada para cálculo do erro médio em cada cadeira do trem de acabamento.

$$
\mathrm{E} \%=\frac{\sum_{\mathrm{i}=1}^{\mathrm{m}}\left|\widehat{\mathrm{y}_{\mathrm{i}}}-\mathrm{y}_{\mathrm{i}}\right|}{\mathrm{m}}
$$

Onde $\widehat{y_{i}}$ e $y_{i}$ se referem respectivamente à carga de laminação calculada e ao valor registrado na planta industrial. $O$ denominador $m$ é o número de medidas realizadas. Em seu trabalho Poliak [20] considera razoável um desvio de até $10 \%$ no cálculo da carga de laminação a quente em um modelo industrial. As causas dos desvios encontrados, podem estar associadas a diferentes fontes como imprecisões na realização das medidas das cargas de laminação industrial, variações

Tabela I. Modelos para previsão da Tensão Média de Escoamento (expressa em MPa)

\begin{tabular}{|c|c|}
\hline Autor & Equação \\
\hline Shida [9] & $\begin{array}{l}\mathrm{TME}_{\text {Shida }}=9,8 I^{*} \sigma_{\mathrm{d}}(\mathrm{C}, \mathrm{T}) \mathrm{f}_{\mathrm{w}}(\varepsilon) \mathrm{f}_{\mathrm{r}}\left(\varepsilon_{\mathrm{s}}\right) ; \sigma_{\mathrm{d}}=0,28 \exp \left(\frac{5}{\mathrm{~T}}-\frac{0,01}{[\mathrm{C}]+0,05}\right) \\
\mathrm{T}=\frac{\mathrm{T}\left[{ }^{\circ} \mathrm{C}\right]+273}{1000} ; \mathrm{f}_{\mathrm{w}}(\varepsilon)=1,3\left(\frac{\varepsilon}{0,2}\right)^{\mathrm{n}}-0,3\left(\frac{\varepsilon}{0,2}\right) \\
\mathrm{n}=0,41-0,07[\mathrm{C}] ; \mathrm{f}_{\mathrm{r}}\left(\varepsilon_{\mathrm{s}}\right)=\left(\frac{\varepsilon_{\mathrm{s}}}{10}\right)^{\mathrm{m}} \\
\mathrm{m}=(-0,019[\mathrm{C}]+0,126) \mathrm{T}+(0,076[\mathrm{C}]-0,05)\end{array}$ \\
\hline Siciliano et al. [12] & $\mathrm{TME}_{\text {Bruna }}=9,81 * \exp \left(\frac{3126+68[\mathrm{C}]+2117[\mathrm{Nb}]+54[\mathrm{Mn}]+152[\mathrm{Mo}]}{\mathrm{T}}\right) \varepsilon^{0,21} \dot{\varepsilon}^{0,13}$ \\
\hline Poliak e Siciliano [II] & $\mathrm{TME}_{\text {Poliak }}=\mathrm{TME}_{\text {Misaka }}(1,09+0,056[\mathrm{Mn}]+4,54[\mathrm{Nb}]+1,21[\mathrm{Ti}]+0,056[\mathrm{Al}]+0,1[\mathrm{Mo}])$ \\
\hline Siciliano et al. [17] & $\mathrm{TME}_{\text {Siciliano }}=9,81 * \exp \left(\frac{2704+3345[\mathrm{Nb}]+220[\mathrm{Mn}]}{\mathrm{T}}\right) \varepsilon^{0,21} \dot{\varepsilon}^{0,13}$ \\
\hline Misaka e Yoshimoto [8] & $\mathrm{TME}_{\text {Misaka }}=9,81^{*} \exp \left(0,126-1,75[\mathrm{C}]+0,594\left[\mathrm{C}^{2}\right]+\frac{2851+2968[\mathrm{C}]-1120\left[\mathrm{C}^{2}\right]}{\mathrm{T}}\right) \varepsilon^{0,21} \dot{\varepsilon}^{0,13}$ \\
\hline Siciliano e Jonas [16] & $\mathrm{TME}_{\text {Siciliano \& Jonas }}=\mathrm{TME}_{\text {Misaka }} *(0,768+0,137[\mathrm{Mn}])$ \\
\hline
\end{tabular}


na composição química do aço, variações dimensionais da tira, heterogeneidades térmicas e uma eventual variação no modelo de previsão da tensão média de escoamento [4].

\section{RESULTADOS}

Os resultados obtidos nas 21 abordagens são apresentados em dois subgrupos, listados na Tabela 2 para os aços C-Mn e na Tabela 3 para os aços microligados ao nióbio. Para previsão da tensão média de escoamento no subgrupo I (aços C-Mn), foram utilizadas as equações de Shida, Poliak \& Siciliano e Siciliano \& Jonas. Neste subgrupo os elementos químicos titânio e nióbio foram considerados iguais a zero.

Para a previsão da TME no subgrupo Il (aços microligados ao nióbio) foram utilizadas as equações de Shida, Poliak \& Siciliano, Siciliano, Marini \& Bruna e Siciliano, Leduc \& Hensger. A primeira análise apresentada a seguir é realizada de forma direta, comparando os valores calculados através dos modelos teóricos e as cargas de laminação registradas no processo industrial, sem a introdução de fatores de ajuste.

São apresentados na Tabela 2 nas colunas FI - F7 os desvios entre as previsões realizadas utilizando as combinações de equações e os valores registrados no laminador industrial. O desvio médio nas sete cadeiras do trem de acabamento (expresso em percentual) é apresentado na coluna $E_{\text {médio }}$. A coluna $\mathrm{f}_{\text {erro }<10 \%}$ refere-se ao percentual de previsões com desvio menor que $10 \%$ em comparação ao valor industrial.

A equação simplificada de Sims obteve o melhor desempenho quando associada às equações para previsão da tensão média de escoamento de Shida e Poliak \& Siciliano. Nesta primeira abordagem a equação de Ekelund obteve o pior resultado, um comportamento similar foi observado por Gorni [4] em seu trabalho.

$\mathrm{Na}$ Figura 2 é apresentada de forma gráfica a dispersão entre as cargas de laminação calculadas para os aços $\mathrm{C}-\mathrm{Mn}$ (sem aplicação de fatores de correção).

Nota-se que a equação de Sims combinada a equação de Shida, utilizada sem os fatores de ajuste, resulta em maiores níveis de dispersão entre os valores calculados e os reais. É observada para este arranjo a tendência em superestimar o valor da carga de laminação nos primeiros passes. Já no último passe de laminação (cadeira F7) nota-se um comportamento oposto, assim a fração média das previsões com dispersões menores que $10 \%$ representaram apenas 34\% dos resultados.

Nota-se na Figura 3 que para os aços microligados ao nióbio a equação de Sims obteve os melhores resultados quando combinada às equações de Shida, Siciliano, Marini \& Bruna e Poliak \& Siciliano (resultados apresentados na Tabela 3). A equação de Sims combinada a equação de Shida (sem fatores de ajuste) tende a subestimar a carga de laminação para os últimos passes, conforme a Figura 4. Para este arranjo de equações, $51 \%$ dos resultados representaram dispersões menores ou iguais a $10 \%$.

$\mathrm{Na}$ comparação direta apresentada na Tabela 2 e Tabela 3, entre os valores registrados no laminador industrial e os resultados das previsões de cargas de laminação, nota-se uma grande dispersão entre os resultados.

Os valores de erro médio registrados no trem acabador e a fração das previsões das cargas de laminação com erro menor ou igual a $10 \%$ verificados na comparação inicial, não se mostraram condizentes ao grau de controle requerido pelo processo industrial.

Desta maneira se justifica a necessidade da adoção de fatores de correção que melhor ajustem os valores das cargas de laminação calculadas utilizando os modelos teóricos as registradas durante o processamento na planta industrial.

Os desvios calculados utilizando a Equação 2 para cada uma das combinações de equações apresentadas na Tabela 2 e Tabela 3 são utilizados como fatores de ajuste.

Estes são adicionados aos novos cálculos para previsão das forças de laminação para ambos subgrupos (aços C-Mn e aços microligados ao nióbio).

Os resultados obtidos após a aplicação dos fatores de ajuste para ambos os aços são apresentados na Tabela $4 \mathrm{e}$ Tabela 5, respectivamente aços C-Mn e aços microligados ao nióbio.

Após a aplicação dos coeficientes de ajuste calculados para os aços C-Mn, as equações de previsão de carga apresentaram erros médios globais variando entre 4,3 e 5,6\%. $\mathrm{Na}$ Figura 3 é verificado um melhor desempenho na previsão das forças de laminação quando o fator de ajuste é aplicado

Tabela 2. Previsão das cargas de laminação no trem de acabamento para os aços C-Mn. Sem aplicação de fatores de correção. Valores expressos em percentuais de erros para cada cadeira

\begin{tabular}{|c|c|c|c|c|c|c|c|c|c|}
\hline Equações & FI & $\mathbf{F 2}$ & F3 & F4 & F5 & F6 & F7 & $\mathbf{E}_{\text {médio }}$ & $f_{\text {erro }<10 \%}$ \\
\hline Sims/Shida & 14,3 & 16,6 & 12,2 & 9,3 & 12,1 & 10,2 & 12,7 & 12,5 & 34 \\
\hline Sims/ Poliak \& Siciliano & 23,2 & 22,4 & 15,7 & 13,0 & 15,0 & 11,5 & 12,7 & 16,2 & 19 \\
\hline Sims/ Siciliano \& Jonas & 23,1 & 24,1 & 34,7 & 39,3 & 36,2 & 41,5 & 80,4 & 39,9 & 2 \\
\hline Orowan-pascoe/ Shida & 25,4 & 24,7 & 17,9 & 12,7 & 13 & 7,5 & 26,4 & 18,2 & 21 \\
\hline Orowan-pascoe/ Poliak \& Siciliano & 33,1 & 30,4 & 21,4 & 16,3 & 15,7 & 8,1 & 28,8 & 22,0 & 17 \\
\hline Orowan-pascoe/ Siciliano \& Jonas & 7,1 & 21,6 & 27,6 & 34,4 & 34,9 & 47,2 & 107,7 & 40,1 & 18 \\
\hline Ekelund/ Shida & 21,8 & 23,5 & 19,3 & 15,5 & 15,1 & 10 & 25,4 & 18,7 & 16 \\
\hline Ekelund/ Poliak \& Siciliano & 30,2 & 29,2 & 22,6 & 19,1 & 17,6 & 19,2 & 24,2 & 23,2 & 12 \\
\hline Ekelund/ Siciliano \& Jonas & 19,6 & 21 & 32 & 38,7 & 40,2 & $5 I, 2$ & 108,4 & 44,4 & 6 \\
\hline
\end{tabular}




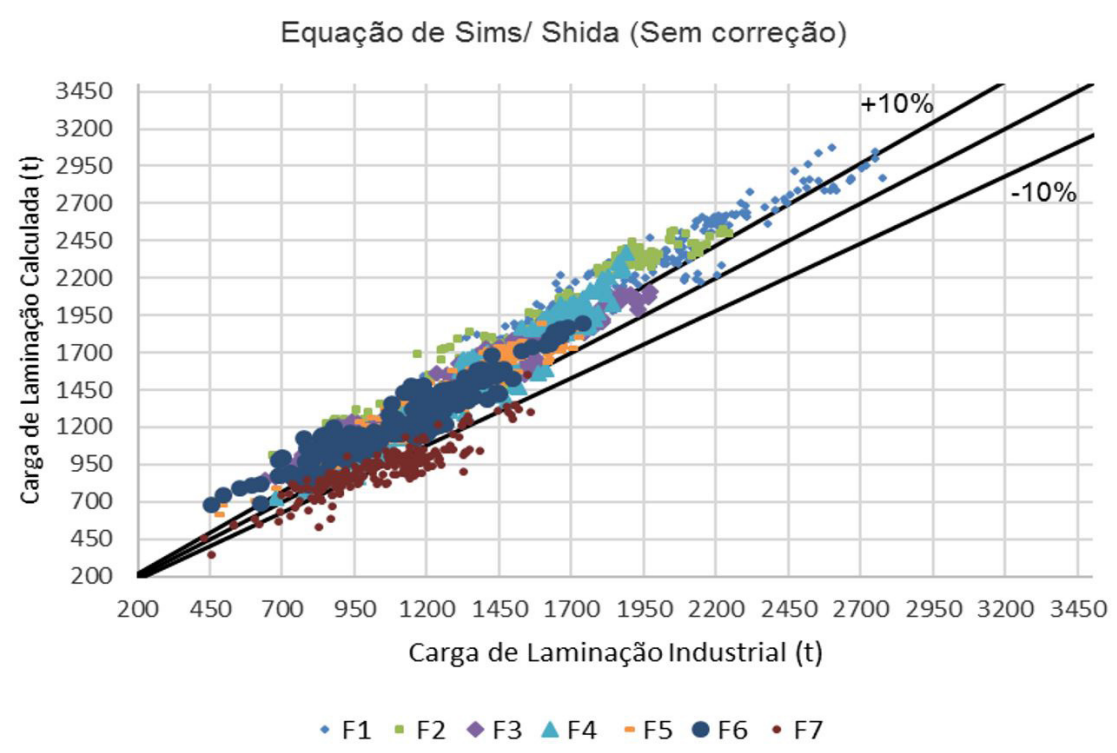

Figura 2. Cargas de laminação calculadas versus as cargas industriais para os aços C-Mn, utilizando a equação de Sims combinada ao modelo de Shida (sem fatores de correção).

Tabela 3. Previsão das cargas de laminação no trem de acabamento para os aços microligados ao nióbio. Sem aplicação de fatores de correção. Valores expressos em percentual

\begin{tabular}{|c|c|c|c|c|c|c|c|c|c|}
\hline Equações & FI & F2 & F3 & F4 & F5 & F6 & F7 & $\mathbf{E}_{\text {médio }}$ & $f_{\text {erro }<10 \%}$ \\
\hline Sims/ Shida & 5,6 & 6,0 & 4,1 & 6,9 & 17,8 & 16,1 & 61,6 & 16,9 & 51 \\
\hline Sims/ Poliak \& Siciliano & 30,1 & 23,9 & 15,9 & 10,8 & 6,6 & 6,1 & 44,0 & 19,6 & 33 \\
\hline Sims/ Siciliano, Marini \& Bruna & 10,9 & 5,7 & 7,3 & 13,8 & 31,4 & 30,3 & 82,6 & 26,0 & 33 \\
\hline Sims/ Siciliano, Leduc \& Hensger & 15,5 & 25,8 & 18,9 & 27,8 & 40,8 & 39,5 & 77,8 & 35,2 & 4 \\
\hline Orowan-Pascoe/ Shida & 21,9 & 17,8 & 9,1 & 7,0 & 14,8 & 16,4 & 74,1 & 23,0 & 28 \\
\hline Orowan-Pascoe/ Poliak \& Siciliano & 42,2 & 33,8 & 24,0 & 16,9 & 6,6 & 6,2 & 55,3 & 26,4 & 20 \\
\hline Orowan-Pascoe/ Siciliano, Marini \& Bruna & 26,4 & 15,6 & 5,1 & 8,2 & 28,1 & 30,7 & 87 & 28,7 & 18 \\
\hline Orowan-Pascoe/ Siciliano, Leduc \& Hensger & 7,3 & 9,1 & 24,2 & 36,0 & 65,0 & 69,0 & 75,3 & 40,8 & 18 \\
\hline Ekelund/ Shida & 13,0 & 15,1 & 9,1 & 5,2 & 15,4 & 21,6 & 88,4 & 24,0 & 33 \\
\hline Ekelund/ Poliak \& Siciliano & 35,8 & 31,8 & 24,1 & 16,5 & 6,5 & 8,7 & 67,9 & 27,3 & 21 \\
\hline Ekelund/ Siciliano, Marini \& Bruna & 18,3 & 13,3 & 4,8 & 7,3 & 28,8 & 36,8 & 82,9 & 27,5 & 22 \\
\hline Ekelund/ Siciliano, Leduc \& Hensger & 5,7 & 11,5 & 24,1 & 36,7 & 66,1 & 76,7 & 86,4 & 43,9 & 18 \\
\hline
\end{tabular}

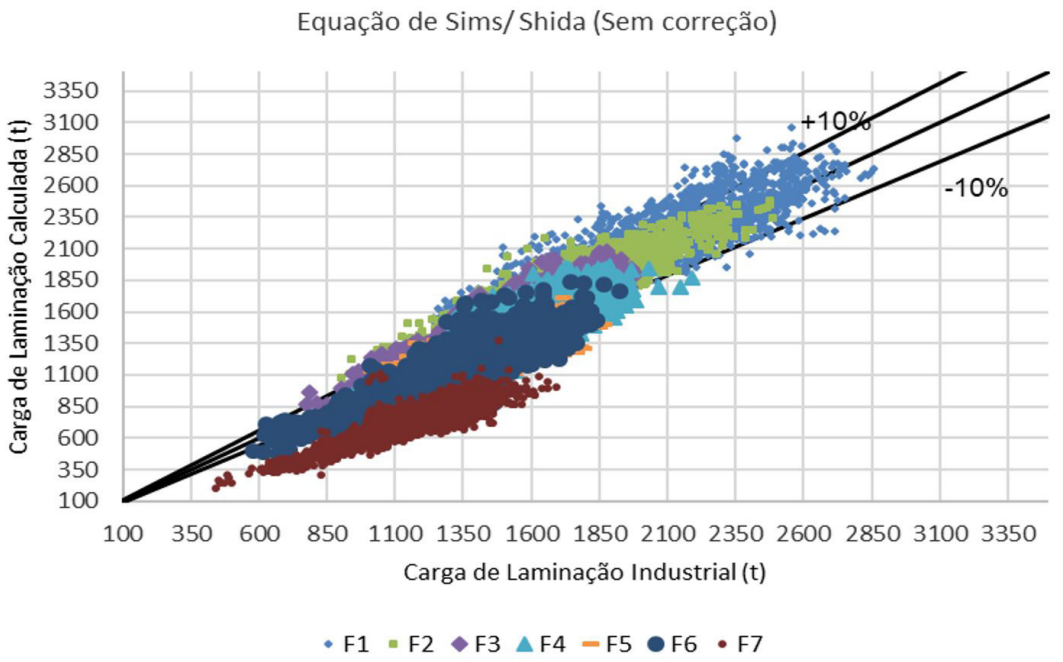

Figura 3. Carga de laminação calculada versus a carga industrial para os aços microligados ao nióbio, utilizando a equação de Sims combinada ao modelo de Shida (sem fatores de correção). 
Dias et al.

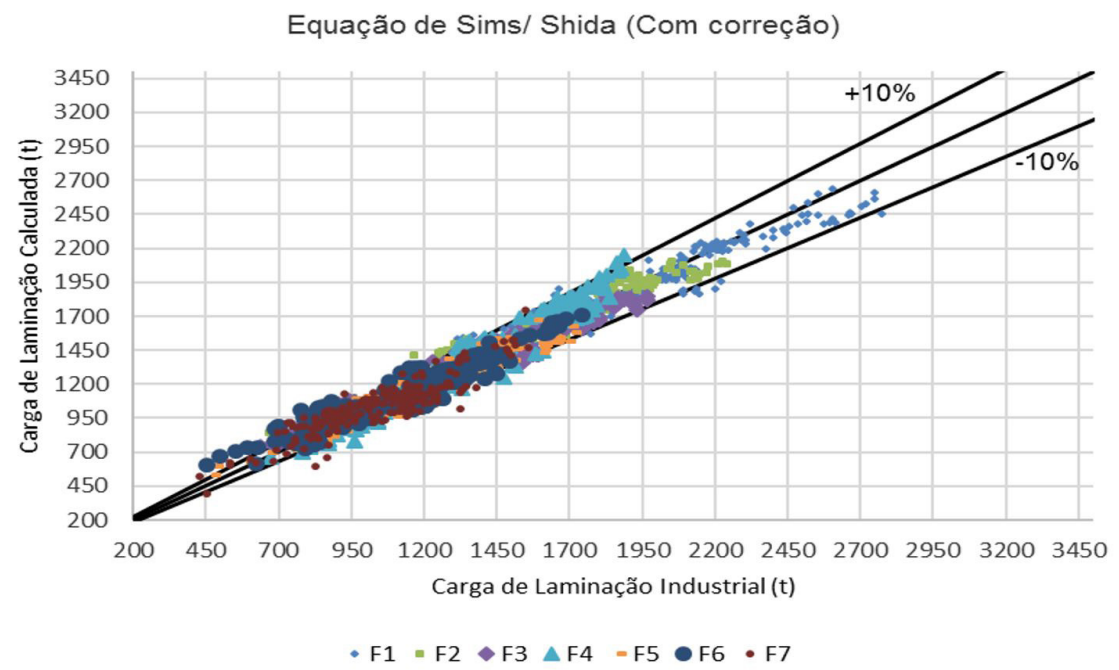

Figura 4. Carga de laminação calculada versus a carga industrial para os aços C-Mn, utilizando a equação de Sims combinada ao modelo de Shida (com fatores de correção).

Tabela 4. Previsão das cargas de laminação no trem de acabamento para os aços C-Mn. Com aplicação de fatores de correção. Valores expressos em percentual

\begin{tabular}{|c|c|c|c|c|c|c|c|c|c|}
\hline Equações & $\mathbf{F I}$ & F2 & F3 & F4 & F5 & F6 & F7 & $\mathbf{E}_{\text {Médio }}$ & $f_{\text {erro }<10 \%}$ \\
\hline Sims/Shida & 3,3 & 3,6 & 3,7 & 4,6 & 4,2 & 4,2 & 6,1 & 4,3 & 87 \\
\hline Sims/ Poliak \& Siciliano & 3,0 & 4,0 & 3,7 & 4,0 & 5,0 & 4,5 & 6,3 & 4,6 & 87 \\
\hline Sims/ Siciliano, Marini \& Bruna & 3,0 & 3,9 & 3,9 & 4,0 & 5,1 & 4,6 & 6,8 & 4,5 & 86 \\
\hline Orowan-pascoe/ Shida & 2,9 & 4,0 & 3,5 & 4,6 & 4,8 & 4,5 & 6,7 & 4,5 & 83 \\
\hline Orowan-pascoe/ Poliak \& Siciliano & 3,5 & 4,4 & 3,9 & 4,1 & 5,4 & 4,4 & 7,2 & 4,8 & 82 \\
\hline Orowan-pascoe/ Siciliano \& Jonas & 3,4 & 4,6 & 3,9 & 4,1 & 5,4 & 4,3 & 7,2 & 4,7 & 81 \\
\hline Ekelund/ Shida & 5,1 & 4,5 & 5,1 & 3,6 & 5,9 & 6,4 & 7,1 & 5,3 & 78 \\
\hline Ekelund/ Poliak \& Siciliano & 4,7 & 5,3 & 4,8 & 4,3 & 6,8 & 5,6 & 7,4 & 5,6 & 75 \\
\hline Ekelund/ Siciliano \& Jonas & 4,4 & 4,6 & 5,3 & 4,8 & 6,6 & 5,5 & 7,1 & 5,5 & 75 \\
\hline
\end{tabular}

Tabela 5. Previsão das cargas de laminação no trem de acabamento para os aços microligados ao nióbio. Com aplicação de fatores de correção. Valor expressos em percentual

\begin{tabular}{|c|c|c|c|c|c|c|c|c|c|}
\hline Equações & FI & F2 & F3 & F4 & F5 & F6 & F7 & $\mathbf{E}_{\text {Médio }}$ & $f_{\text {erro }<10 \%}$ \\
\hline Sims/Shida & 5,4 & 4,1 & 4,7 & 6,2 & 5,9 & 6,0 & 6,2 & 5,5 & 78 \\
\hline Sims/ Poliak \& Siciliano & 4,2 & 5,1 & 3,6 & 6,9 & 7,3 & 7,0 & 6,0 & 5,7 & 76 \\
\hline Sims/ Siciliano, Marini \& Bruna & 4,1 & 5,7 & 3,8 & 7,2 & 6,4 & 6,0 & 6,0 & 5,6 & 71 \\
\hline Sims/ Siciliano, Leduc \& Hensger & 4,1 & 5,6 & 4,8 & 6,8 & 6,3 & 5,9 & 6,3 & 5,7 & 78 \\
\hline Orowan-Pascoe/ Shida & 4,3 & 4,5 & 3,9 & 7,2 & 6 & 5,7 & 6,1 & 4,6 & 77 \\
\hline Orowan-Pascoe/ Poliak \& Siciliano & 5,8 & 6,3 & 4,4 & 7,5 & 6,9 & 6 & 6 & 6,1 & 72 \\
\hline Orowan-Pascoe/ Siciliano, Marini \& Bruna & 5,9 & 6,8 & 4,7 & 8,2 & 6,6 & 5,6 & 6 & 6,3 & 74 \\
\hline Orowan-Pascoe/ Siciliano, Leduc \& Hensger & 6,2 & 6,9 & 4,4 & 8 & 6,5 & 5,5 & 6,7 & 6,3 & 73 \\
\hline Ekelund/ Shida & 5,5 & 4,8 & 4,5 & 5,7 & 5,7 & 6,4 & 6,8 & 5,6 & 75 \\
\hline Ekelund/ Poliak \& Siciliano & 4,5 & 6,3 & 4,2 & 6,1 & 7,1 & 6,5 & 7 & 6,0 & 73 \\
\hline Ekelund/ Siciliano, Marini \& Bruna & 5 & 7,4 & 4,3 & 7,2 & 8 & 6,2 & 4,9 & 6,1 & 74 \\
\hline Ekelund/ Siciliano, Leduc \& Hensger & 4,8 & 6,5 & 4,3 & 6,4 & 6,3 & 6,5 & 8,6 & 6,2 & 73 \\
\hline
\end{tabular}

aos conjuntos de equações. A assertividade da combinação entre a equação de Sims e o modelo para previsão da TME de Shida, passa de $34 \%$ para $87 \%$ das previsões, com dispersões menores ou iguais a $10 \%$.

Nota-se na Tabela 5 que com a aplicação dos coeficientes de ajustes calculados para os aços microligados ao nióbio, o erro médio global para as sete cadeiras do trem de acabamento se manteve entre 4,6 e 6,3\%.

Após aplicação dos coeficientes de ajuste verifica-se na Figura 5 o aumento da assertividade das previsões, onde $78 \%$ dos resultados passaram a apresentar erros iguais ou inferiores a $10 \%$. 
Equação de Sims/Shida (Com correção)

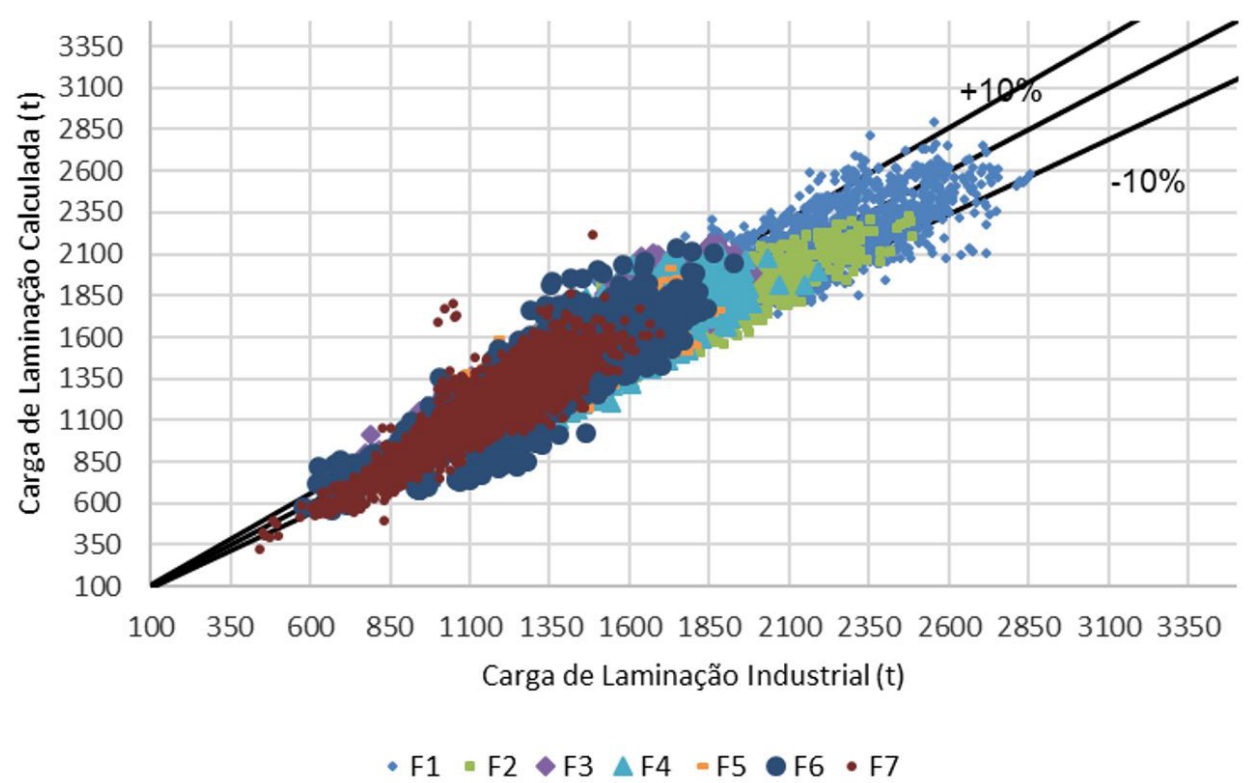

Figura 5. Carga de laminação calculada versus a carga industrial para os aços microligados ao nióbio, utilizando a equação de Sims combinada ao modelo de Shida (com fatores de correção).

\section{DISCUSSÕES}

Os conjuntos de equações teóricas apresentados nas Tabelas 2 e 3, permitiram a previsão das cargas de laminação e cálculo das tensões medias de escoamento sem a necessidade da realização de numerosos testes experimentais e recursos computacionais sofisticados. Porem os resultados iniciais (sem aplicação de fatores de ajuste) apresentaram elevada dispersão entre os valores registrados no laminador industrial e os calculados.

$\mathrm{Na}$ condição inicial o emprego da equação de Shida combinada as equações de Sims, Orowan-Pascoe e Ekelund, resultou em um menor desvio médio global entre as cargas de laminação calculadas e os valores industriais para ambos subgrupos. Segundo Dimatteo [13] o melhor desempenho do modelo de Shida pode estar associado à dependência da taxa de deformação ao expoente temperatura.

As equações para previsão das forças de laminação de Sims, Orowan-Pascoe e Ekelund apresentaram o melhor nível de desempenho quando combinadas à equação para previsão da TME de Poliak \& Siciliano em comparação aos resultados obtidos com a equação de Siciliano, Leduc \& Hensger.

melhor desempenho das equações de Sims, Orowan-Pascoe e Ekelund quando combinadas a equação de Poliak \& Siciliano, pode estar associada ao maior número de elementos de liga que são levados em consideração na determinação da tensão média de escoamento.

Vale observar que as equações utilizadas para as previsões das tensões médias de escoamento, consideram apenas a recristalização estática. Desta maneira o efeito da ocorrência de eventuais transformações microestruturais como a recristalização dinâmica, metadinâmica, a precipitação de nitretos e carbonetos são adicionados aos conjuntos de equações por meio da utilização dos fatores de ajustes. Estes foram calculados através do desvio médio apresentado por cada cadeira do trem acabador e são apresentados nas Tabelas 2 e 3.

Após a introdução dos fatores de ajustes calculados para o subgrupo I (aços C-Mn), os arranjos de equações passaram a presentar erros meios globais variando entre 4,3 e 5,6\%. Com a aplicação dos fatores de ajuste o subgrupo II (aços microligados ao nióbio) apresentou significativa melhora, com erros médios globais variando entre 4,6 e 6,3\%.

\section{CONCLUSÕES}

É apresentada neste trabalho uma análise de diferentes arranjos de modelos teóricos para o cálculo das cargas de laminação para aços C-Mn e aços microligados ao nióbio, com base em 2676 dados de processamentos em uma planta industrial. Para os aços C-Mn, em uma comparação direta entre as cargas de laminação calculadas e os valores reais registrados na planta industrial, as equações de Shida, Poliak \& Siciliano combinadas à equação de Sims apresentaram os melhores níveis de desempenho, seguidas pelas equações de Orowan-Pascoe e Ekelund. Após a aplicação dos coeficientes de ajuste calculados para os aços C-Mn, as equações de previsão de carga apresentaram erros médios globais variando entre 4,3 e $5,6 \%$. 
Para os aços microligados ao nióbio a equação de Sims combinada a equação de Shida apresentou os melhores resultados em ambas as comparações (com e sem ajuste). Após a aplicação dos fatores de ajuste as equações apresentaram erros médios globais no trem de acabamento variando entre 4,6 e $6,3 \%$.

\section{Agradecimentos}

A Universidade Federal Fluminense e ao PPGEM, pela permissão e apoio na realização desse trabalho. Aos Engenheiros Sidney Ferreira da Silva e Otavio Luiz Cardoso de Souza pelas contribuições técnicas e suporte ao trabalho.

\section{REFERÊNCIAS}

I Devadas C, Baragar D, Ruddlel G, Samarasekera V, Hawbolt EB. The thermal and metallurgical state of steel strip during hot rolling: part ii. factors influencing rolling loads. Metallurgical Transactions. A, Physical Metallurgy and Materials Science. 1991;22:335-349.

2 Orowan E. The calculation of roll pressure in hot and cold flat rolling. Proceedings of the Institution of Mechanical Engineers. 1943; 150: 140-167.

3 Wusatowski Z. Fundamentals of rolling. Oxford: Pergamon; 1969.

4 Gorni A, Silva M. Comparação entre os modelos para o cálculo de carga na laminação a quente industrial. Tecnologica em Metalurgia, Materiais e Mineração. 20I2;9:197-203.

5 Sims RB. The calculation of roll force and torque in hot rolling mills. Proceedings - Institution of Mechanical Engineers. 1954;168:191-214.

6 Ekelund S. Analysis of factors influencing rolling pressure and power consumption in the hot rolling of steel. Vol. 93. [S.I.]: [s.n.]; 1933. p. 39.

7 Hodgson P. Models of the recrystallisation behaviour of c-mn and $\mathrm{nb}$ microalloyed steels during hot working processes. Materials Forum. 1993; 17:403-408.

8 Misaka Y, Yoshimoto T. Formulation of mean resistance of deformation of plain carbon steel at elevated temperature. Journal of the Japan Society for Technology of Plasticity. 1967-1968;8:4I4-422.

9 Shida S. Effect of carbon content, temperature and strain rate on compressive flow stress of carbon steel. Hitachi Research Lab Report. 1974; 1:1416-1425.

10 Kirihata A, Siciliano F Jr, Maccagno TM, Jonas JJ. Mathematical modelling of mean flow stress during the hot strip rolling of multiply-alloyed medium carbon steels. ISIJ International. 1998;38(2): I87-195.

II Poliak El, Siciliano F. Hot deformation behavior of Mn-Al and Mn-Al-Nb steels. In: Materials Science \& Technology Conference; 2004; New Orleans, USA. Loisiana: AIST; 2004. pp. 39-45.

12 Siciliano F, Marini O, Bruna RG. The effect of chemical composition on the hot deformation resistance during hot strip rolling of microalloyed steels processed at the Sidor hot strip mill. In: Super High Strength Steels Conference Proceedings, Associazione Italiana di Metallurgia; 2005 November; Roma. Milão: Associazione Italiana di Metallurgia; 2005.

I3 Dimatteo A, Vannucci M, Colla V. Prediction of mean flow stress during hot strip rolling using genetic algorithms. ISIJ International. 2014;54:171-178.

14 Shida S. Empirical formula of flow stress of carbon steels: resistance to deformation of carbon steels at elevated temperature. Japan Society for Technology Plasticity. 1969;10:610-617.

15 Shafiei E, Goodarzi N, Dehghani K, Tehrani AS. A new constitutive equation to predict single peak flow stress curves at high temperatures: a comprehensive study on different steels. Canadian Metallurgical Quarterly. 2017;56(I): 104112.

16 Siciliano F, Jonas JJ. Mathematical modeling of the hot strip rolling of Nb microalloyed, Cr-Mo and Plain C-Mn Steels. Metallurgical and Materials Transactions. A, Physical Metallurgy and Materials Science. 2000;31:5II.

17.Siciliano F, Leduc LL, Hensger KH. The hot deformation resistance and microstructure evolution during processing of microalloyed steels in thin slab casting/direct rolling process. In: Czech Metallurgical Society. Proceedings of the Steelsim-Simulation and Modelling of Metallurgical Processes in Steelmaking; 2005 October 25-27; Brno, Czech Republic. Ostrava, Vitkovice: Czech Metallurgical Society; 2005.

18 Alghamdi F. Mathematical Modeling of Mean Flow Stress (MFS) during Hot Strip Rolling for HSLA steels [thesis]. Montreal: McGill University; 2014. 
19 Roberts WL. Tribological considerations in the hot-rolling of low-carbon steels. Lubrication Engineering. 1977;33:575-580.

20 Poliak EI, Shim MK, Kim GS, Choo WY. Application of linear regression analysis in accuracy assessment of rolling force calculations. Metals and Materials. 1998;4:1047-1056.

Recebido em: 3 Maio. 2018

Aceito em: 7 Fev. 2019 